\title{
The genus Holothele Karsch, 1879: the identity of the type species (Mygalomorphae, Theraphosidae)
}

\author{
José Paulo Leite GUADANUCCI \\ Departamento de Zoologia, Instituto de Biociências, \\ Universidade Estadual Paulista, avenida 24A 1515, \\ 13506-900 Rio Claro-SP (Brazil) \\ joseguadanucci@gmail.com \\ Carlos PERAFÁN \\ Sección Entomología, Facultad de Ciencias, \\ Universidad de la República, Iguá 4225, Montevideo (Uruguay) \\ caperafanl@gmail.com \\ Dayana VALENCIA-CUÉLLAR \\ Grupo de Investigación en Insectos Neotropicales, \\ Universidad del Magdalena, Santa Marta, Magdalena (Colombia) \\ daysofia1024@gmail.com
}

Published on 30 June 2017

KEY WORDS

Ischnocolinae, spider, tarantula, South America, new records, new synonyms.

MOTS CLÉS

Ischnocolinae, araignée, tarentule,
are du Sud Amérique du Sud signalisations nouvelles,
synonymes nouveaux.
urn:Isid:zoobank.org:pub:945FE92F-7AD3-4EE3-8B1E-A8787644EA1D

Guadanucci J. P. L., Perafán C. \& Valencia-Cuéllar D. 2017. - The genus Holothele Karsch, 1879: the identity of the type species (Mygalomorphae, Theraphosidae). Zoosystema 39 (2): 263-271. https://doi.org/10.5252/z2017n2a5

\section{ABSTRACT}

The genus Holothele Karsch, 1879 has a confusing taxonomic history, mainly due to a imprecise and outdated generic diagnosis, perpetuated since its original description. In this work, we propose a new diagnosis for the genus, redescribe the type species Holothele recta Karsch, 1879 and propose a few taxonomic changes. Holothele longipes is here considered as a senior synonym of Holothele recta Karsch, 1879, Stichoplastus sanguiniceps F. O. P.-Cambridge, 1898, Dryptopelmides rondoni Lucas \& Bücherl, 1972, Dryptopelmides ludwigi Strand, 1907. We also provide updated geographic distribution records for Holothele longipes and propose the revalidation of Scopelobates Simon, 1903.

\section{RÉSUMÉ}

Le genre Holothele Karsch 1879: l'identité de l'espèce type (Mygalomorphae, Theraphosidae).

Le genre Holothele Karsch, 1879 a une histoire taxonomique assez confuse, essentiellement due à sa diagnose imprécise et dépassée, qui se perpétue depuis sa description originale. Dans ce travail, nous proposons une nouvelle diagnose pour le genre, redécrivons l'espèce type Holothele recta Karsch, 1879 et proposons quelques changements taxonomiques. Holothele longipes est ici considéré un synonyme senior de Holothele recta Karsch, 1879, Stichoplastus sanguiniceps F. O. P.-Cambridge, 1898, Dryptopelmides rondoni Lucas \& Bücherl, 1972 et Dryptopelmides ludwigi Strand, 1907. Nous présentons aussi des signalisations nouvelles pour Holothele longipes, et proposons de revalider Scopelobates Simon, 1903. 


\section{INTRODUCTION}

The genus Holothele Karsch, 1879 (Theraphosidae) can be considered a major taxonomic confusion, mainly due to the lack of a precise diagnosis. As a result, several species have been improperly included. The genus, originally established as monotypic, was described based on a female from Caracas, Venezuela, named $H$. recta Karsch, 1879 as type-species. The inclusion of all other species currently within the genus is due to subsequent transfers or descriptions, along with other species that have already been removed from Holothele in the past years (Guadanucci et al. 2007; Guadanucci \& Weinmann 2014, 2015).

Raven (1985) considered Holothele as a senior synonym of Schismatothele Karsch, 1879, Euthycaelus Simon, 1889, Hemiercus Simon, 1903, Scopelobates Simon, 1903 and Dryptopelmides Strand, 1907. Although Raven (1980) brought up the possibility of Stichoplastus Simon, 1889 as junior synonym of Holothele, he did not establish this change. As a result of Raven's proposals, the genus Holothele included eleven species at that time. Subsequentely, Rudloff (1997) proposed the following combinations: the Caribbean H. culebrae (Petrunkevitch, 1929), H. denticulata (Franganillo, 1930) and H. shoemakeri (Petrunkevitch, 1926) were transferred to Holothele from Ischnocolus Ausserer, 1871; H. incei (F. O. Pickard-Cambridge, 1898) from Hapalopus Ausserer, 1875; H. longipes (L. Koch, 1875) from Chaetopelma; H. vellardi Rudloff, 1997 and H. rondoni (Lucas \& Bücherl, 1972) from Cyclosternum Ausserer, 1871. Moreover, Rudloff (1997) also revalidated Schismatothele and Euthycaelus. The revalidation of Euthycaelus was not followed in Platnick's catalog (2013) since it did not provide a detailed diagnosis for the genus. Finally, Rudloff (1997) also considered the genus Stichoplastus as junior synonym of Holothele. Recently, Guadanucci \& Weinmann (2014) removed the genera Schismatothele and Euthycaelus from the synonym with Holothele.

Regarding its familial relationships, Raven (1980) removed the genus Holothele from Dipluridae Simon, 1889 and placed it in Theraphosidae Thorell, 1869. Raven (1985) later included it in Theraphosinae subfamily, from where it was finally transferred to Ischnocolinae Simon, 1892 by Pérez-Miles et al. (1996). Guadanucci (2014) considered Holothele as closely related to representatives of Trichopelma Simon, 1888, Reichlingia Rudloff, 2001 and Ischnocolus within Ischnocolinae sensu stricto. Holothele currently comprises 10 species from northern South America, southern Central America and the West Indies (World Spider Catalog 2016).

In this paper, based on the examination of type material and extensive collection specimens, we present a redescription of the type species of the genus, propose a few taxonomic rearrangements, report color variation of specimens from distinct localities and present a distribution map for the species.

\section{MATERIAL AND METHODS}

Specimens from the following institutions were examined. Abbreviations, cities, countries and curator are as follows:

AMNH American Museum of Natural History, New York, (N. I. Platnick);
BMNH British Museum of Natural History, London (J. Beccaloni);

IBSP Instituto Butantan, São Paulo (D. B. Battesti);

ICN Instituto de Ciencias Naturales, Universidad Nacional de Colombia, Bogotá (E. Flórez);

MNHN Muséum national d'Histoire naturelle, Paris (C. Rollard);

MZSP Museu de Zoologia da Universidade de São Paulo, São Paulo (R. Pinto-da-Rocha);

NMW Naturhistorisches Museum Wien, Vienna, (J. Gruber);

RMNH Nationaal Natuurhistorische Museum ("Naturalis"), Leiden (L. Willemse);

ZMB Museum für Naturkunde der Humboldt-Universität, Berlin (J. Dunlop);

ZMUH Universität von Hamburg, Zoologisches Institut und Zoologisches Museum, Hamburg (H. Dastych);

ZSM Zoologische Staatssammlung, Munich (R. Melzer)

Collection

coll. DW

Private collection, D. Weinmann, Stuttgart.

\section{MEASUREMENTS}

All measurements are in millimetres and were taken with a micrometer ocular lens. The length of legs segments was measured between the joints in dorsal view. The length and width of carapace, eye tubercle, labium and sternum are the maximum values obtained. The total body length includes the chelicerae and opistosoma but not the spinnerets. Number and disposition of spines follows the terminology of Petrunkevitch (1925).

All drawings were made with drawing tube (Leica MZ APO). Pictures were taken with a Nikon Coolpix 5400 attached to the microscope. The spermathecae were cleared with carnation oil and illustrated from dorsal view. Left palpal bulbs were removed from the cymbium and illustrated in dorsal, pro- and retrolateral views. The setae of the male tibia I were removed to expose the tibial spur.

The geographic coordinates and altitude data (meters above sea level: $m$ a.s.l.) were referenced by GPS, Datum WGS84, or determined using the Instituto Geográfico Agustín Codazzi Gazetter (http://www.igac.gov.co/digeo/app/index.html) and Global Gazetter version 2.3 (http://www.fallingrain.com/ world/index.html). The distribution map was produced using SimpleMappr (Shorthouse 2010).

\section{SYSTEMATICS}

Classe ARACHNIDA Lamarck, 1801

Order ARANEAE Clerck, 1757

Suborder MYGALOMORPHAE Pocock, 1892

Family THERAPHOSIDAE Thorell, 1869

Genus Holothele Karsch, 1879

Holothele Karsch, 1879: 544. — Simon 1892: 182. — Raven 1980: 254. — Rudloff 1997: 7.

Stichoplastus Simon, 1889: 208; 1892: 141; (type species: Stichoplastus ravidus Simon, 1889, from Corosal, San Esteban, Venezuela). 
Dryptopelmides Strand, 1907: 18 (type species: Dryptopelmides rondoni Lucas \& Bücherl, 1972, from Puerto Cabelo, Venezuela).

TyPE SPECIES. - Holothele recta Karsch, 1879, by monotypy.

DiagnOSIS. - Representatives of the genus are distinguished by the combination of the following features: absence of urticating setae; tarsus IV pseudosegmented; tarsal claws with a median row of teeth; labium trapezoid, with approximately 90 cuspules or fewer; sigillae close to sternal margin.

\section{EMENDED DESCRIPTION}

Chelicerae without rastellum. Males with intercheliceral intumescense. Cephalic region slightly raised. Eye tubercle weakly raised, wider than long. Anterior eye row procurved, posterior recurved. Thoracic fovea, deep, slightly recurved. Labium trapezoid, with 50-90 cuspules. Maxilla with produced anterior lobe, several cuspules on the inner angle (more than 80). Labium-sternal sigilla well marked, oval, mounds raised. Anterior sternal sigillae marginal, posterior sternal sigilla submarginal. Metatarsal scopula present on all legs, I totally occupied, II distal 3/4, III around distal half, IV less than distal half. Tarsal scopula I-IV divided by a band of stout setae. Tarsus IV straight (contra Raven 1985) and pseudosegmented. Clavate tarsal trichobothria in two rows, interspersed with filiform trichobothria. Proximal lateral faces of tibia with thickened trichobothria, dorsal face with filiform. Retrolateral scopula on femur IV absent. Stridultory setae absent. Superior tarsal claws with single median row of teeth, inferior tarsal claws absent, claw tufts well developed. Urticanting setae absent. Posterior lateral spinnerets three-segmented, long, apical segment digitiform. Cymbium longer than wide, bilobed. Palpal bulb with elongate embolus, increasingly filiform towards tip, without keels, subtegulum short. Male tibial apophysis present, formed by two branches, metatarsus I bends laterally to the retrolateral branch. Spermathecae formed by two elongate, narrow base, and flat receptacles, swollen distally, and with or without lobes on the inner ventral face.

Holothele longipes (L. Koch, 1875) (Figs 1-5)

Chaetopelma longipes L. Koch in Ausserer, 1875: 174, pl. 6, figs 20, 21.

Holothele recta Karsch, 1879: 544. — Rudloff 1997: 10, n. syn.

Stichoplastus ravidus Simon, 1889: 208. — Rudloff 1997: 10, figs 1, 2.

Stichoplastus sanguiniceps F. O. P.-Cambridge, 1898: 895, n. syn.

Dryptopelmides rondoni Lucas \& Bücherl, 1972: 234, figs 1-4, n. syn.

Dryptopelmides ludwigi Strand, 1907: 19, n. syn.

Holothele longipes - Rudloff 1997: 9.

Holothele rondoni - Rudloff 1997: 10.

TYPE MATERIAL. - Holotype 9 of Holothele recta (ZMB 457), from Caracas, Venezuela, Gollmar 1879, examined. - Syntypes, $10^{7}$, $2 \%$ and 2 juveniles of Stichoplastus ravidus (MNHN-AR-AR4625), from San Esteban, Venezuela, examined (note: there are several places named San Esteban in Venezuela. Simon (1889) explored within the limits of the provinces of Carbobo and Guzman Blanco, that includes to date Aragua, Guárico, Miranda, Nueva Esparta and Federal District. He further mentions that San Esteban is two hours from Puerto Cabello, Carabobo, and that he explored the forest at the banks of San Esteban River, near Puerto Cabello). Holotype o" of Chaetopelma longipes (BMNH 19.9.18.5728), from Puerto Cabello, Carabobo, Venezuela, examined. - Holotype + of Stichoplastus sanguiniceps (BMNH 1895.5.3.1), from Trinidad, J. H. Hart, examined. - Holotype o" and paratype + of Dryptopelmides rondoni (IBSP 4090), from lauaretê, Amazonas, Brazil, A. R. Hoge, F. Saliba and N. P. Santos, Jan.1971, examined. - Holotype + of Dryptopelmides ludwigi, from Puerto Cabello, Venezuela, not examined, presumably lost.

Additional material examined. - Bolívia. La Paz, Coroico, 4532 m a.s.l., $10^{7}$, NMW.

Brazil. Amazonas: Iauaretê: $10^{\text {đa }} 1$ juv., IBSP 4090; Manaus: Rio Jacaré, 1 o", IBSP 168490, 17.XI.1996, N. Gordon; Santa Maria: 1 , MZSP 21504; 1 ㅇ, MZSP 21507; Mato Grosso: Apiacás: $10^{7}$, MZSP 18046, 10-15.II.1997, G. Skuk, V. X. Da Silva.

Colombia. Amazonas: La Pedrera, Quebradon, El Ayo, 1³5’S, 69³1'W, 85 m a.s.l., $10^{\circ}$, ICN-Ar 6852, V.2002, J. Pinzón. Atlántico: Barranquilla, $10^{\circ} 57^{\prime} 50^{\prime \prime} \mathrm{N}, 74^{\circ} 47^{\prime} 47^{\prime \prime W}, 30 \mathrm{~m}$ a.s.l., $10^{\prime \prime}$, ICN-Ar 042, X.1963. - Bolívar: Turbaco: Finca El Huerto, 10²2’19”N, $75^{\circ} 20^{\prime} 58.8$ ”W, $102 \mathrm{~m}$ a.s.l., 1 \% , ICN-Ar 8049; Jardín Botánico, $10^{\circ} 21^{\prime} 14^{\prime \prime} \mathrm{N}, 75^{\circ} 25^{\prime} 41.5^{\prime \prime W}, 131 \mathrm{~m}$ a.s.l., 1 \%, ICN-Ar 8053, 7.VII.2015, A. Sabogal \& C. Cantor. - Cesar: Valledupar, Ecoparque Los Besotes: Monte Puma, $10^{\circ} 34^{\prime} 0.6^{\prime N}$, 73¹7’2”W, 950 m a.s.l., 1 \% , ICN-Ar 8051, 19.VII.2015, Grupo Carbio; Los Zainos, 1 o", ICN-Ar 8052, 18.VII.2015, Grupo Carbio. - Cundinamarca: La Calera, El Manzano, PNN Chingaza, Río Blanco, Palacio, Laguna Blanca, 441'24”N, 7351'21"W, 2930 m a.s.l., 1 o', IavH, 19-21. XI.2003, E. González; Mesitas del Colegio, Santa Cecilia, Finca El Naranjal, $4^{\circ} 45^{\prime} 28^{\prime \prime N}, 73^{\circ} 24^{\prime 2} 27^{\prime \prime W}, 1000$ m a.s.l., $10^{\prime \prime}$, ICN-Ar 8047 , 23-30.XII.2011, A. García \& J. Diaz. - Guaviare: San José de Guaviare, Playa Guio, $2^{\circ} 34^{\prime} 22^{\prime} \mathrm{N}, 72^{\circ} 38^{\prime} 45^{\prime} \mathrm{W}, 213 \mathrm{~m}$ a.s.l.: $20^{\prime \prime}$, ICN-Ar 8048, 28.X.2012, N. Téllez; 1 ơ, ICN-Ar 8050, 10.X.2013, E. Flórez. - Meta: Acacias, La Esmeralda, Finca Versalles, $3^{\circ} 25^{\prime} 47^{\prime} \mathrm{N}$, $74^{\circ} 5^{\prime} 3^{\prime}$ W, 515 m a.s.l., 1 juv., 21.IX.2004, E. Flórez; Guamal, $10^{\prime \prime}$, coll. DW KS286; Puerto Gaitán, Carimagua, 4ำ18'48”N, 724'54”W, 160 m a.s.l., 22.IV.2012, D. Martínez; San Juan de Arama, Reserva La Macarena, 3²2'12”N, 7352'22”W, 450 m a.s.l., 1 juv., 18.I.1992, F. Guzmán \& S. Espinoza. - Norte de Santander: La Donjuana: $10^{7}$, MZSP 28381, 19.XI.2001, D.Weinmann; Pamplonita: 1 우, MZSP 28380, 2.X.1998, D. Weinmann; 1 ○’, MZSP 28382; 1 ㅇ, MZSP 28384; 1 \%, MZSP 28383, 2.X.1998, D. Weinmann; San Jose de Avila: $10^{\circ}$, SMF 10101. — Santander: Bolívar, Portachuelo, Verbeo, Cueva La Olla, 559'22”N, 7346'13”W, 2050 m a.s.l., 2 juv., ICN-Ar 6960, 19.VII.2008, M. Hoyos; Cabrera, Cueva del Indio de Dońa Joaquina, 6 $35^{\prime} 36^{\prime} \mathrm{N}, 73^{\circ} 14$ '52”W, $1000 \mathrm{~m}$ a.s.l., ICN-Ar 6957: 2 \%, ICN-Ar 6958, 1 ㅇ, 14.VII.2007, Grupo de Bioespeleología; Villanueva, Agua Fría, Cueva del Nitro, 640'19”N, 73¹0'29”'W, 1500 m a.s.1., 1 \% , 6 juv., ICN-Ar 6961, 13.VII.2007, Grupo de Bioespeleología; Zapatoca, Cueva, 648'54”N, 73¹6'6”W, 1 , ICN-Ar 8054, V.2008, C. Perafán \& F. Pérez Miles. — Sucre: La Garita: 19 juv., ZMUH, W. Fritsche, 26.IX.1912; San Marcos,

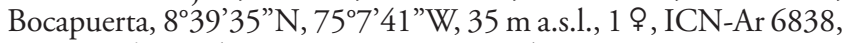
19.X.2004, Estudiantes Sistemática Animal. — Vaupés: Taraira, Bajo Río Apaporis, Lago Taraira, Estación Biológica Caparú, 0²9’38”N, 6940'1”W, 200 m a.s.l., 2 o", ICN-Ar 2003, J. Pinzón.

French Guiana. Montsinery-Tonnegrande: Emerald Jungle Village, 1 ơ, IBSP 168520, 447'5”N, 52²5'21”W, 9.IV.1999, R. C. West. Peru. Loreto: Río Momón, Amazon Camp: 1 \%, IBSP 168508; 1 우, IBSP 168524; 1 Ơ, IBSP168523, 8.XI.1993, R. C. West; 1 juv., IBSP 168521, 6.X.1993, W. Lamar; 1 오. IBSP 168525, II.1993, T. Mason. 


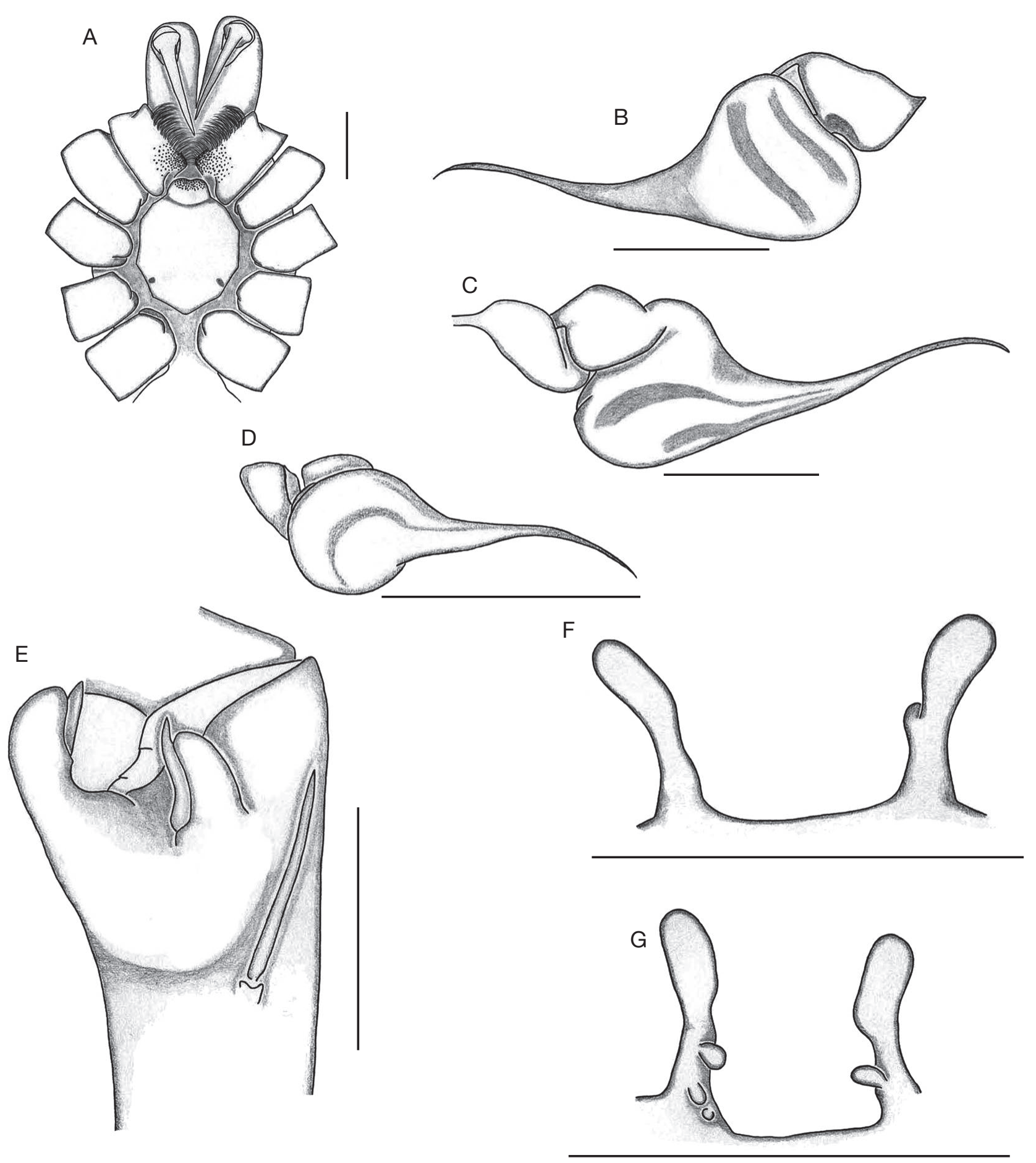

FIG. 1. - Holothele longipes (L. Koch, 1875): A-E, ơ (MNHN 9501); A, cephalotorax, ventral view; B, palpal bulb, retrolateral view; C, palpal bulb, prolateral view; D, palpal bulb, ventral view; E, ơ tibial apolhysis, prolateral-ventral view; F, G, o (MNHN 950): F, spermathecae receptacles, dorsal view; G, spermathecal receptacles, ventral view. Scale bars: $1 \mathrm{~mm}$.

Surinam. Wanica: Lelydorp, 1 , AMNH, 1.XII.1938, Geiskes. Trinidad \& Tobago. Arima: 1 , AMNH, 23.VII.1979, R. Mendez; 1 juv., AMNH, 23.VII.1979, L. N. Sorkin. - Caparo: 1 \% AMNH, 15.III.1910, P. B. Whelpley; 2 9, AMNH, 16.II.1910, P. B. Whelpley. — Port-of-Spain: 1 , AMNH, 2.VII.1965, Erik N. Kjellesvig-Waering. - Tamana: Deep Park Tamana Cave: 1 ,
BMNH 1971/296, 19.I.1968, Darlington; 1 , 1 juv., BMNH 1971/231, 13.XII.1968, Darlington; 1 o", BMNH 1971/298, 5.III.1968, Darlington; $10^{7}$, BMNH 1971/293, 21.X.1968, Darlington; 1 O’, BMNH 1971/294, 16.XII.1968, Darlington; 2 juv., BMNH 1971/297, 16.X.1968, Darlington. — Toco: 1 ơ $^{\prime \prime}$ AMNH, 14.II.1965, Erik N. Kjellesvig-Waering. 

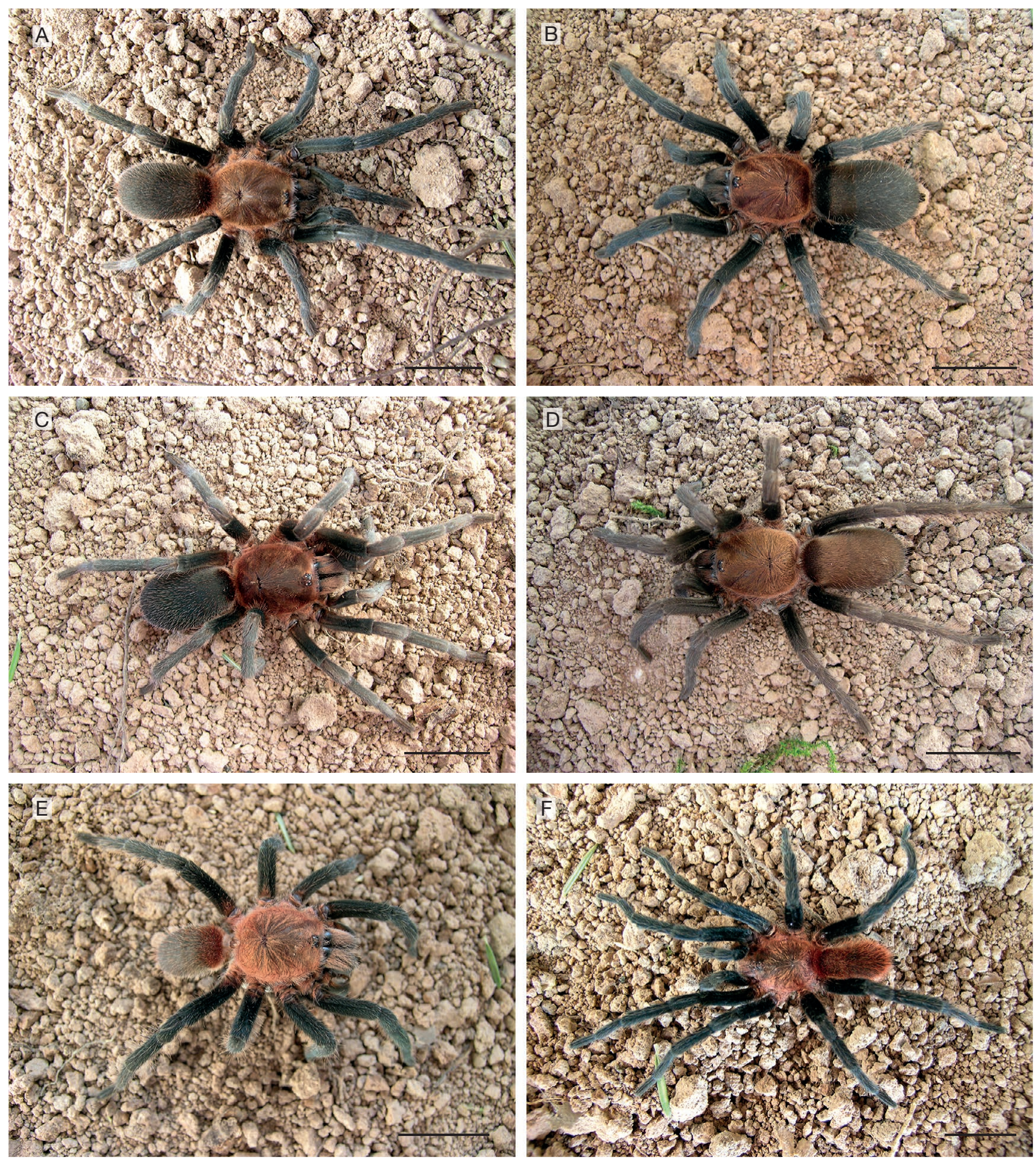

FIG. 2. - Holothele longipes (L. Koch, 1875), showing color variation: A, , from Caracas, Venezuela; B, $ᄋ$, from Trinidad; C, $\$$, from Meta, Colombia; D, $९$, from Puerto Cabello, Venezuela; E, o, from Pamplonita, Colombia; F, ơ, from Pamplonita, Colombia. Scale bars: 10 mm. Photographs: José P. Guadanucci.

Venezuela. Amazonas: San Pedro: 1 ơ, IBSP 168491, 2.X.1991, R. C. West. - Aragua: Parque Nacional Henri Pittier: 10 , NRS, 12-30.XI.1997, T. Pape; Maracay: 3 o", ZSM, 1939, C. Vogl. Anzoategui: Romerito: $10^{\prime \prime}$, MZSP 28387; 2 \% MZSP 28388-28389; 10 ơ, MZSP 28385. — Distrito Federal: Caracas: 1 우, coll. DW KS73. - Bolívar: Las Trincheras: 1 \%, ZMUC 595; 1 ơ, 1 juv.,
ZMUC 243, C. Levinsen, 18.XII.1991. - Carabobo: Puerto Cabello: 1 \%, ZMUH, Lievers, X.1993; 1 \%, NMW, R. Riemann, 1-5.II.1989; 1 ơ $^{7}$ IBSP 168506, 5.X.1998, H. Bauer; Rancho Grande, $10^{\prime \prime}$, MZSP 28386. — Falcon: San Esteban: $10^{7}, 2$, 1 juv., MNHN; San Luis: 1 \% , MZSP 28390, $11^{\circ} 07^{\prime} 66.5^{\prime \prime} \mathrm{N}$, 6940’66.4”W, 21.IX.2002, D. Weinmann \& F. Pribik. — Mé- 

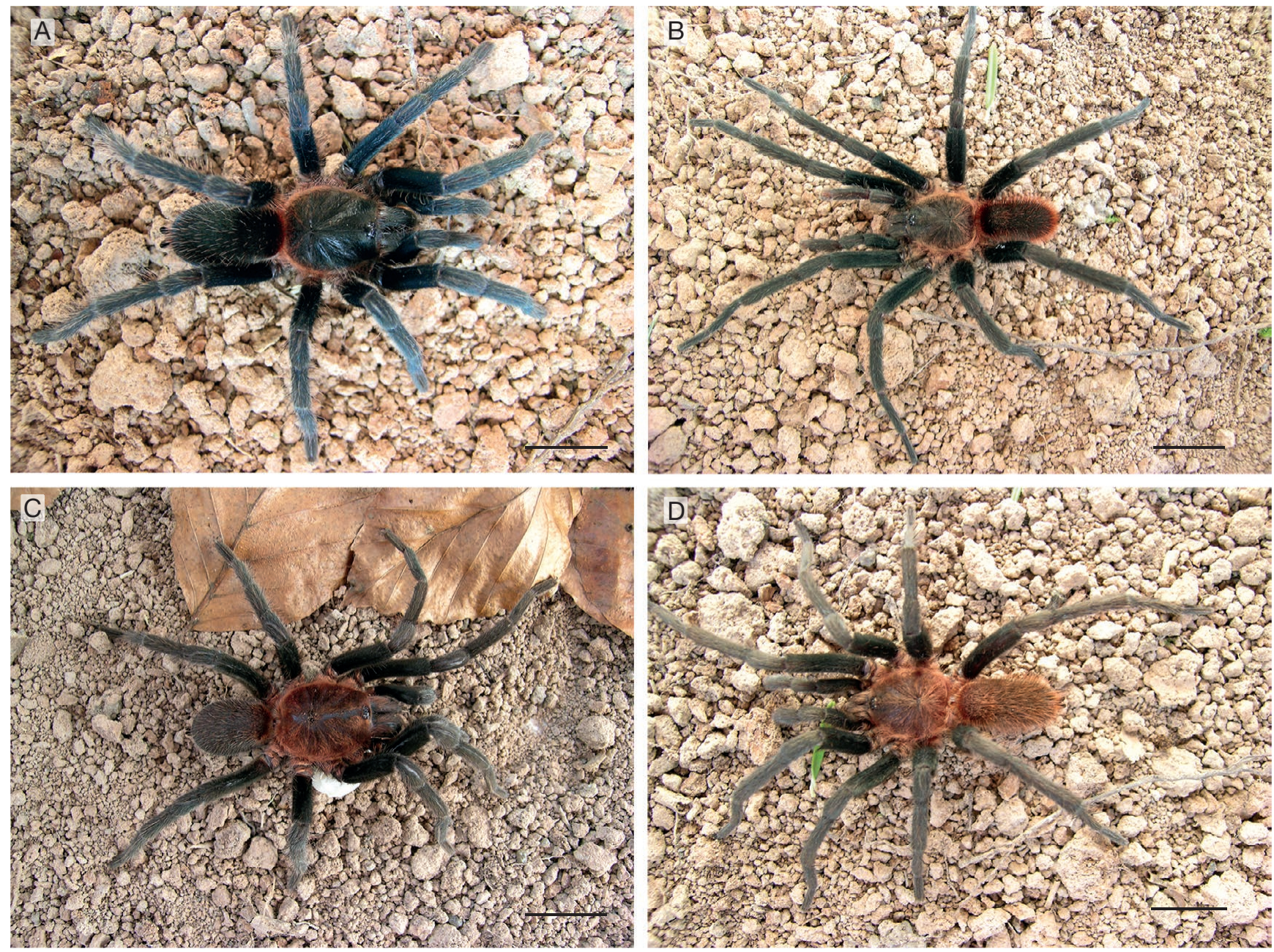

FIG. 3. - Holothele longipes (L. Koch, 1875), showing color variation: A, ๆ, from Romerito, Venezuela; B, ơ, from Romerito, Venezuela; C, ९, from Táchira, Venezuela; D, ơ, from Táchira, Venezuela. Scale bars: $10 \mathrm{~mm}$. Photographs: José P. Guadanucci.

rida: Mérida: 7 o", 3 \%, 1 juv., MNHN-AR-AR4626; 1 o", BMNH 03.7.1.130; 1 0", BMNH 1905.3.31.24-26; La Azulita: $10^{\circ}$, MZSP 28391, 26.IX.2002, D. Weinmann; 1 \%, MZSP 28392, 26.IX.2002, D. Weinmann; Parque Nacional Sierra de la Culata, 3000 m a.s.l., $10^{7}$, 1 , BMNH 03.7.1.128-129; 1 0", BMNH 05.3.31.61-70. — Monagas: Caripito: 3 ㅇ, AMNH, 15-31.III.1942, NY Zool. Soc.; 1 웅 AMNH, No. 4220; 1 \%, AMNH, 10-20.V.1945, W. Beebe et al. — Táchira: San Juan de Colón: $10^{7}$, MZSP 28393, 0807’52.8”N 70¹4'10.4”W, 23.IX.2002, D. Weinmann, F. Pribik; 1 ㅇ, MZSP 28394; 1 오, MZSP 28395.

Type Locality. - Venezuela. Puerto Cabello, Estado Carabobo.

Diagnosis. - Females can be distinguished by the spermathecae receptacula elongate, with a narrow base, slightly swollen at the apical end, bearing or not lobes on the inner ventral face, these lobes may vary in size and number (Figs 1F, G; 4A-F). Males are distinguished by the morphology of the palpal bulb, which is long, thin, and slightly curved (Fig. 1B-D), by the morphology of the tibial apophysis, formed by two branches, retrolateral with a tapering tip (Fig. 1E), and by the presence of a intercheliceral intumescense. Males differ from the small species from the Caribbean (H. sulfurensis Maréchal, 2005, H. culebrae, $H$. denticulata and $H$. shoemakeri) by the absence of a twisted embolus with small keels. Females differ from those species by the shape of spermathecae, which bear lobes (except the specimens inhabiting caves).
Coloration. - Males and females with legs and palps black; carapace covered with setae that may vary from red to black, setae around carapace red; abdomen covered with setae that vary from black to red; chelicerae covered with setae of the same color as those on carapace (Figs 2, 3).

Distribution. $-H$. longipes occupies a wide geographic range of northern South America. It is distributed from the $0 \mathrm{~m}$ a.s.l. in Trinidad and Tobago, along the Caribbean coast of Colombia and Venezuela, and the Atlantic coast of Surinam, to above $2000 \mathrm{~m}$ a.s.l. on the eastern cordillera of Colombia. It is registered for Bolivia, Peru, Brazil, Colombia, Venezuela and Suriname (Fig. 5).

NATURAL HISTORY. - This species has managed to conquer different environments within a wide altitudinal gradient. It inhabits humid forests of the Brazilian and Colombian Amazon, Andean forests and grasslands, and coastal savannahs of Colombia and Venezuela. They have been found especially under rocks and logs, and also within human constructions. So its ecological plasticity is evident. Additionally, some specimens have been found inside the caves from Santander, Colombia. These specimens are only few females and juveniles, and show no troglomorphic adaptations. Therefore, it is possible to assume that these specimens are using caves as temporary refuge. 

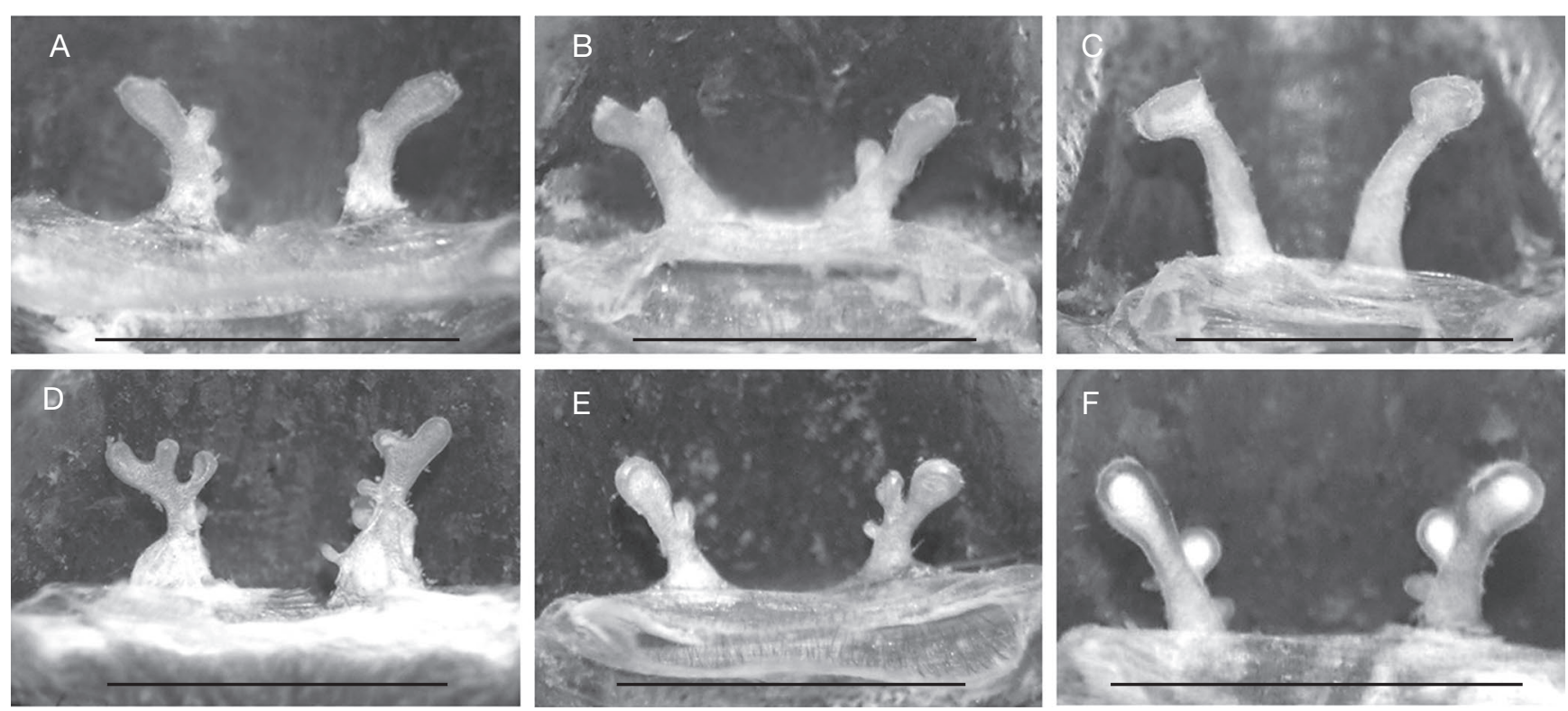

FIG. 4. - Holothele longipes (L. Koch, 1875), spermathecal morphology variation, dorsal view: A, o from Romerito, Aragua, Venezuela; B, o from Colón, Táchira, Venezuela; C, o from Altamira, Venezuela; D, o from Pamplonita, Santander, Colombia; E, o from La Azulita, Táchira, Venezuela; F, o from Santa Maria, Amazonas, Brazil. Scale bars: $1 \mathrm{~mm}$.

\section{EMENDED DESCRIPTION}

\section{Female (holotype 9 of $\mathrm{H}$. recta)}

Total length 16. Carapace: length 6.7; width 5.6. Eye tubercle: length 0.9; width 1.3. Labium: length 0.8; width 1.3. Sternum: length 3; width 2.9. Cheliceral basal article with 14-15 teeth. Labium nearly as wide as long bearing more than 50 cuspules. Maxilla with more than 100 cuspules. Sternum rounded, sigilla one diameter from margin. Thoracic furrow slightly recurved. Palpal and legs segments lengths: palp: femur 3.9/ patella 2.4/ tibia 2.9/ tarsus 2.8/ total 12. Leg I: femur 4.4/ patella $2.5 /$ tibia $3.6 /$ metatarsus $2.9 /$ tarsus $2.2 /$ total 15.6 . II: 4.7/ 2.8/3.7/ 3.2/ 2.4/ 16.8. III: 4.4/ 2.4/ 3.2/ 4/ 2.4/ 16.4. IV: $5.7 / 2.7 / 5.1 / 6 / 3 / 22.5$. Spines: tarsi without spines. Palp: femur (d) 0-0-p1, tibia (v) 1-2-ap3, (p) 0-1-0. Legs: I: femur (d) 0-0-p1, tibia (v) 0-1-ap1, metatarsus (v) 1-1-ap1. II: femur (d) 0-0-p1, tibia (v) 0-1-ap2, (p) 1-1-0, metatarsus (v) 1-1-ap1. III: femur (d) 0-0-2, patella (p) 1, (r) 1, tibia (v) 2-2-ap3, (p) 1-1-0, (r) 1-1-0, metatarsus (v) 3-1-2+ap3, (p) 1-1-1, (r) 1-1-1. IV: femur (d) 0-0-2, tibia (v) 2-2-ap3, (p) $0-1-0,(r) 1-1-1$, metatarsus (v) 2-2-2+ap3, (p) 0-1-1, (r) 1-1-1. Spermathecae formed by two receptacula longer than wide, without lobes. Scopula on metatarsi: I totally occupied, II $3 / 4$ occupied, III half occupied and IV less than half occupied. Tarsal scopula I-IV divided. Tarsal claws with a median row of small teeth, clavate trichobothria in tow rows, divided by a central row of thin long setae. Tarsus IV cracked. Eyes: anterior row procurved, posterior slightly recurved, clypeus absent (note: the holotype specimen described above is a juvenile $\%$, as already noted by Rudloff (1997), because it does not have well sclerotized genital opening and the divison of the anterior tarsal scopula is very wide. Moreover, the size of the specimen is very small, unlike the adult representatives of this species. Although it presents a spermathecae, it is well known that juvenile theraphosids have undeveloped receptacula (Schiapelli \& Gerschman de Pikelin 1962). We, therefore, present a description based on the type material of Stichoplastus ravidus (junior synonym of $H$. longipes), in order to provide a description based on an adult specimen.

\section{Male (MNHN-AR-AR4625, Syntype adult male of}

Stichoplastus ravidus)

Total length 28.8. Carapace: length 12.6; width 10.9. Eye tubercle: length 1.4; width 1.7. Labium: length 1.2; width 2.1. Sternum: length 5.2; width 4.7. Cheliceral basal article with 15 teeth, intercheliceral intumescence present. Labium nearly as wide as long bearing fewer than 90 cuspules. Maxilla with more than 100 cuspules. Sternum rounded, sigilla one diameter from margin. Thoracic furrow slightly recurved. Palpal and legs segments lengths: Palp: femur 6.8/ patella 4.2/ tibia 5.8/ cymbium 2/ total 18.8. Leg I: femur 11.7/ patella 6.2/ tibia 10.8/ metatarsus 8.8/ tarsus 6/ total 43.5. II: $10.9 / 5.6 /$ 9.6/ 9/ 5.5/ 40.6. III: 10/ 4.8/ 8.2/ 10.8/ 5.2/ 39. IV: $12.4 /$ 5.1/ 11.2/ 14.8/ 7/ 50.5. Spines: Tarsi without spines. Palp: femur (d) 0-1-p1, patella (p) 1, tibia (v) 2-1-0, (p) 0-1-1. Leg I: femur (d) 0-2-1, tibia (v) 2-2-ap1, (p) 1-1-2, metatarsus (v) 1-1-ap1. II: femur (d) 0-1-2, patella (p) 1, tibia (v) 2-2-ap3, (p) 1-1-0, metatarsus (v) 2-1-ap 1, (p) 0-1-0. III: femur (d) 3-3-4, patella (p) 1, (r) 1, tibia (v) 3-3-ap3, (p) 1-1-0, (r) 1-10 , metatarsus (v) 2-2-2+ap3, (p) 1-1-1, (r) 1-1-1. IV: femur (d) 3-4-4, patella (p) 1, (r) 1, tibia (v) 2-1-ap3, (p) 1-1-1, (r) 1-1-2, metatarsus (v) 3-3-ap3, (p) 1-1-1, (r) 1-1-2. Male palpal bulb with long, thin, and slightly curved embolus. Tibial apophysis formed by two branches. Retrolateral long, tapered, bearing a spine on its midlength. Prolateral short bearing spines on the base. Metatarsus I bends laterally to the prolateral branch. Scopula on metatarsi: I totally occupied, 


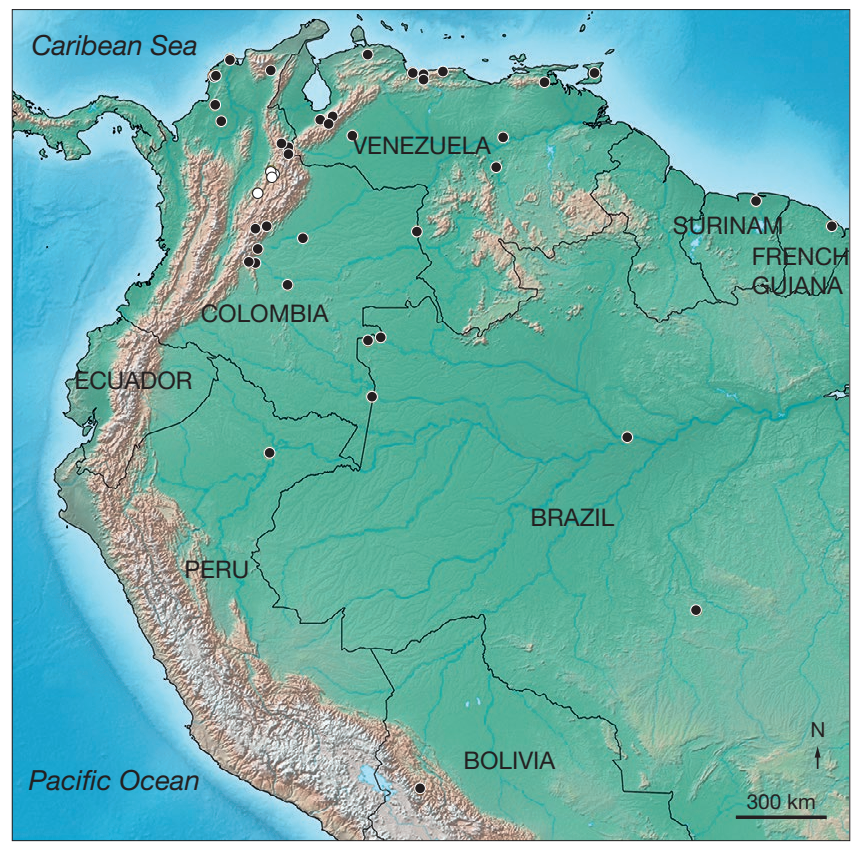

FIG. 5. - Geographical distribution records for Holothele longipes (L. Koch, 1875). Symbols 0 indicate specimens found in caves.

II 3/4 occupied, III nearly more than half occupied, IV less than half occupied. Scopula on tarsi I-IV divided. Tarsal claws with a median row of small teeth, clavate trichobothria in two rows, divided by a central row of long and thin setae. Tarsus IV cracked. Eyes: anterior row procurved, posterior slightly recurved, clypeus absent.

Female (MNHN-AR-AR4625 Syntype adult of of Stichoplastus ravidus)

Total length 46.8. Carapace: length 14.8; width 12. Eye tubercle: length 2.3; width 1.7. Labium: length 1.8 ; width 2.5 . Sternum: length 5.8; width 5.6. Cheliceral basal article with 17-19 teeth. Labium nearly as wide as long, bearing more than 80 cuspules. Maxilla with more than 100 cuspules. Sternum rounded, sigilla one diameter from margin. Thoracic fovea slightly recurved. Palpal and legs segments lengths: palp: femur 8.1/ patella $4.8 /$ tibia $6.1 /$ tarsus 5.4 / total 24.4. Leg I: femur $11.1 /$ patella $6.9 /$ tibia $9.1 /$ metatarsus $7.5 /$ tarsus $4.9 /$ total 39.5. II: 9.7/ 6/ 7.8/ 7.3/ 4.8/ 35.6. III: 9.4/ 5.2/ 6.9/ 9.4/ 4.8/ 30.9. IV: $12.4 / 6 / 10.8 / 14.2 / 5.5 / 48.9$. Spines: Tarsi without spines. Palp: femur (d) 0-0-p1, tibia (v) 1-2-ap3, (p) 1-1-0. Leg I: femur (d) 0-0-p1, tibia (v) 1-1-ap3, metatarsus (v) 1-1-ap1. II: femur (d) 0-0-p1, tibia (p) 1-1-0, (v) 1-1-ap3, metatarsus (v) 1-1-ap1. III: femur (d) 0-0-2, patella (p) 1, (r) 1, tibia (v) 1-2-ap3, (p) 1-1-0, (r) 1-1-0, metatarsus (v) 3-3ap3, (p) 1-1-1, (r) 1-1-1. IV: femur (d) 0-0-2, patella (r) 1, tibia (v) 2-3-ap3, (p) 1-1-0, (r) 1-1-0, metatarsus (v) 2-4-ap3, (p) 1-1-1, (r) 1-1-1. Spermathecae formed by two receptacula longer than wide bearing small lobed on the inner basal portion (note: in some specimens, these lobes may be located on the ventral side of the receptaculum). Scopula on metatarsi: I totally occupied, II $3 / 4$ occupied, III more than half occupied,
IV less than half occupied. Tarsal scopula I-IV divided. Tarsal claws, clavate trichobothria, tarsus IV and eyes as in male.

\section{MISPLACED SPECIES}

Genus Scopelobates Simon, 1903 nom. rest.

Scopelobates Simon, 1903: 928.

Holothele - Raven, 1985: 159. — Pérez-Miles et al. 1996: 60.

TyPE SPECIES. - Scopelobates sericeus Simon, 1903, comb. rest., by monotypy.

\section{Scopelobates sericeus Simon, 1903}

Scopelobates sericeus Simon, 1903: 928.

Holothele sericea - Raven 1985: 159. — Perez-Miles et al. 1996 : 60. — Rudloff 1997: 11, fig. 8-11.

TYPe MATERIAL. - Holotype 9 (MNHN-AR-AR5032) from Ins. Santo Domingo, Dominic Republic, examined.

\section{REMARKS}

The examination of the holotype revealed the presence of a conspicuous dorsal abdominal patch of urticating setae type I and III, which clearly makes it a representative of the subfamily Theraphosinae. Interestingly, this feature had not been noticed before. This species is very similar to representatives of the genus Stichoplastoris Rudloff, 1997. The type specimen does not possess any stridulating setae, and therefore, the two Dominican genera Phormictopus Pocock, 1901 and Cyrtopholis Simon, 1892 could not accommodate it. However, without a comprehensive examination of the type material of the species currently included in the genus Stichoplastoris, any taxonomic rearrangement should not be proposed. Therefore, as a useful step towards revised nomenclature we remove this species from Holothele and revalidate Scopelobates. In this way, a taxonomic survey including Theraphosinae species from West Indies would clarify this issue.

\section{DISCUSSION}

Within Theraphosidae, the position of the genus Holothele, as well as some other Theraphosidae genera (e.g., Dolichothele Mello-Leitão, 1923; Chaetopelma Ausserer, 1871; Heterothele Karsch, 1879; Ischnocolus Ausserer, 1871; Plesiophrictus Pocock, 1899) have been under debate for the last three decades, since Raven (1985) revised the whole infraorder Mygalomorphae. Guadanucci (2014), using morphological characters in a phylogeny including spiders from all subfamilies of Theraphosidae placed Holothele rondoni new synonym of $H$. longipes in a group named Ischnocolinae sensu stricto. Other species formerly included in Holothele fell into a group named Schismatothelinae Guadanucci, 2014, which led to some taxonomic transfers and descriptions by Guadanucci \& Wienmann $(2014,2015)$. 
A previous revision of the genus Holothele, by Rudloff (1997), considered as important characters to distinguish among species the following features: shape of fovea, eyes disposition, spermathecae morphology and spination. Having examined exstensive material from various localities, we did not recognize any pattern to warrant species distinction, due to high level of morphological variation. Spination shows great variation in number and disposition even between right and left legs. Concerning the spermathecae, we also observed some variation, but within a morphological pattern, in which the lobes vary in number among the specimens examined (Fig. 4).

Considering all taxonomic synonymies proposed here, the genus Holothele now comprises five species: H. longipes; H. culebrae (Petrunkevitch, 1929); H. denticulata (Franganillo, 1930); H. shoemakeri (Petrunkevitch, 1926) and H. sulfurensis Maréchal, 2005. Holothele longipes is a widespread species with records in northern South America (Fig. 5), and the remaining species are present in different Caribbean islands (as listed above, respectively from Puerto Rico, Cuba, United States, Virgin Islands and Guadeloupe). These latter Caribbean species share with Holothele longipes the trapezoidal shape of labium, number and disposition of maxillary and labial cuspules, presence of maxillary heel, cracked tarsi and tibial thickened trichobothria (Guadanucci 2014). According to the description of H. sulfurensis Maréchal, 2005, and the examination of additional specimens attributable to the genus from Antígua, Saint John, Saint Martín, Grenada, West Antígua, Hans Lock Island and Guana Island (deposited at AMNH, MCZ and RMNH, pers. obs. JPLG), all Caribbean specimens differ from $H$. longipes by the twisted embolus of palpal bulb and the presence of a ventral notch at the base of metatarsus I in males and by the non-lobed, flat spermathecal receptacles in females.

\section{Acknowledgements}

We are very grateful to the curators of zoological collections mentioned for allowing us the revision of the material. Thanks also to Rick West and Martin Gamache for sending specimens from French Guiana and Peru, and Dirk Weinmann for his great contribution in granting access to specimens from Colombia and Venezuela. CP is grateful to the Agencia Nacional de Investigación e Innovación (ANNI), Uruguay, by the postgraduate grant POS_NAC_2011_1_3624. We also thank two anonymous reviewers for valuable suggestions and critiques.

\section{REFERENCES}

AusSERER A. 1875. — Zweiter Beitrag zur Kenntniss der ArachnidenFamilie der Territelariae Thorell (Mygalidae Autor). Verhandlungen der Kaiserlich-Königlichen Zoologisch-Botanischen Gesellschaft in Wien 25: 125-206.

GUADANUCCI J. P. L. 2014. — Theraphosidae phylogeny: relationships of the "Ischnocolinae" genera (Araneae, Mygalomorphae). Zoologica Scripta 43 (5): 508-518. https://doi.org/10.1111/zsc. 12065

GuadanuCCI J. P. L. \& WeinManN D. 2014. - The spider genera Euthycaelus Simon and Schismatothele Karsch (Mygalomor- phae, Theraphosidae). Zootaxa 3795 (3): 275-288. https://doi. org/10.11646/zootaxa.3795.3.3

GUADANUCCI J. P. L. \& WeINMANN D. 2015. — Description of Neoholothele gen. nov. (Araneae, Theraphosidae, Schismatothelinae). Studies on Neotropical Fauna and Environment 50 (3): 221-228. https://doi.org/10.1080/01650521.2015.1110309

Guadanucci J. P. L., Lucas S. M., Indicatti R. P. \& YAMaMOTO F. U. 2007. - Description of Guyruita gen. nov. and two new species (Ischnocolinae, Theraphosidae). Revista Brasileira de Zoologia 24 (4): 991-996. https://doi.org/10.1590/S010181752007000400015

KARSCH F. 1879. - Arachnologische Beitrage. Zeitschrift für die gesammten Naturwissenschaft 52: 534-562.

LUCAS S. \& BÜCHERL W. 1972. — Redescrição de Dryptopelmides Strand 1907 (Araneae, Orthognatha, Theraphosidae, Ischnocolinae) e descrição de Dryptopelmides rondoni sp. n. Memórias do Instituto Butantan 36: 233-241.

Pérez-Miles F., Lucas S. M., Da Silva Jr. P. I. \& Bertani R. 1996. - Systematic revision and cladistic analysis of Theraphosinae (Araneae: Theraphosidae). Mygalomorph 1: 33-68.

Petrunkevitch A. 1925. - Arachnida from Panama. Transactions of the Connecticut Academy of Arts and Sciences 27: 51-248.

PiCKARD-CAMBridge F. O. 1899. - On new species of spiders from Trinidad, West Indies. Proceedings of the Zoological Society of London 66 (4, for 1898): 890-900, Pl. LIV. [published in April 1899, after Duncan 1937].

RAVEN R. J. 1980. - The evolution and biogeography of the mygalomorph spider family Hexathelidae (Araneae, Chelicerata). Journal of Arachnology 8: 251-266.

RAVEN R. J. 1985. - The spider infraorder Mygalomorphae (Araneae): Cladistics and systematics. Bulletin of the American Museum of Natural History 182: 1-180.

RudLOFF J. P. 1997. - Revision der Gattung Holothele Karsch, 1879 nebst Aufstellung einer neuen Gattung Stichoplastoris gen. nov. (Araneae, Theraphosidae) und Wiedereinsetzung einiger weiterer Gattungen der Mygalomorphae. Arachnologische Magazin 5 (2): 1-19.

Schiapelli R. D. \& Gerschman De Pikelin B. S. 1962. Importancia de las espermatecas en la sistematica de las arañas del Sudordem Mygalomorphae (Araneae). Physis 64: 69-75.

Shorthouse D. P. 2010. - SimpleMappr, an online tool to produce publication-quality point maps. [Retrieved from http:// www.simplemappr.net]

SimON E. 1889. — Voyage de M. E. Simon au Venezuela (décembre 1887-avril 1888). Arachnides. 4e memoire. Annales de la Société Entomologique de France 6 (9): 169-220.

SimON E. 1892. - Histoire naturelle des araignées (Tome 1). Paris, Roret, 552 p. https://doi.org/10.5962/bhl.title.51973

SimON E. 1903. - Histoire naturelle des araignées (Tome 2). Paris, Roret, 1102 p. https://doi.org/10.5962/bhl.title.51973

STRAND E. 1907. - Aviculariidae und Atypidae des Kgl. Naturalienkabinetts in Stuttgart. Jahreshefte des Vereins für vaterländische Naturkunde in Württemberg 63: 1-100.

ThORELl T. 1869. - On European spiders. Part I. Review of the European genera of spiders, preceded by some observations on zoological nomenclature. Nova Acta Regiae Societatis Scientiarum Upsaliensis 7 (3): 1-108.

World SPider CATAlog. 2016. — World Spider Catalog. Natural History Museum of Bern, online at http://wsc.nmbe.ch, version 17.5, accessed on October, 16th, 2016. 


\section{Downloaded From: https://bioone.org/journals/Zoosystema on 01 Aug 2019}

\section{Terms of Use: https://bioone.org/terms-of-use $\quad$ Access provided by Universidad de la Republica}

